\title{
Brackets, superalgebras and spectral gap
}

\section{Consuelo Martínez \& Efim Zelmanov}

São Paulo Journal of Mathematical Sciences

ISSN 1982-6907

São Paulo J. Math. Sci.

DOI 10.1007/s40863-019-00128-5

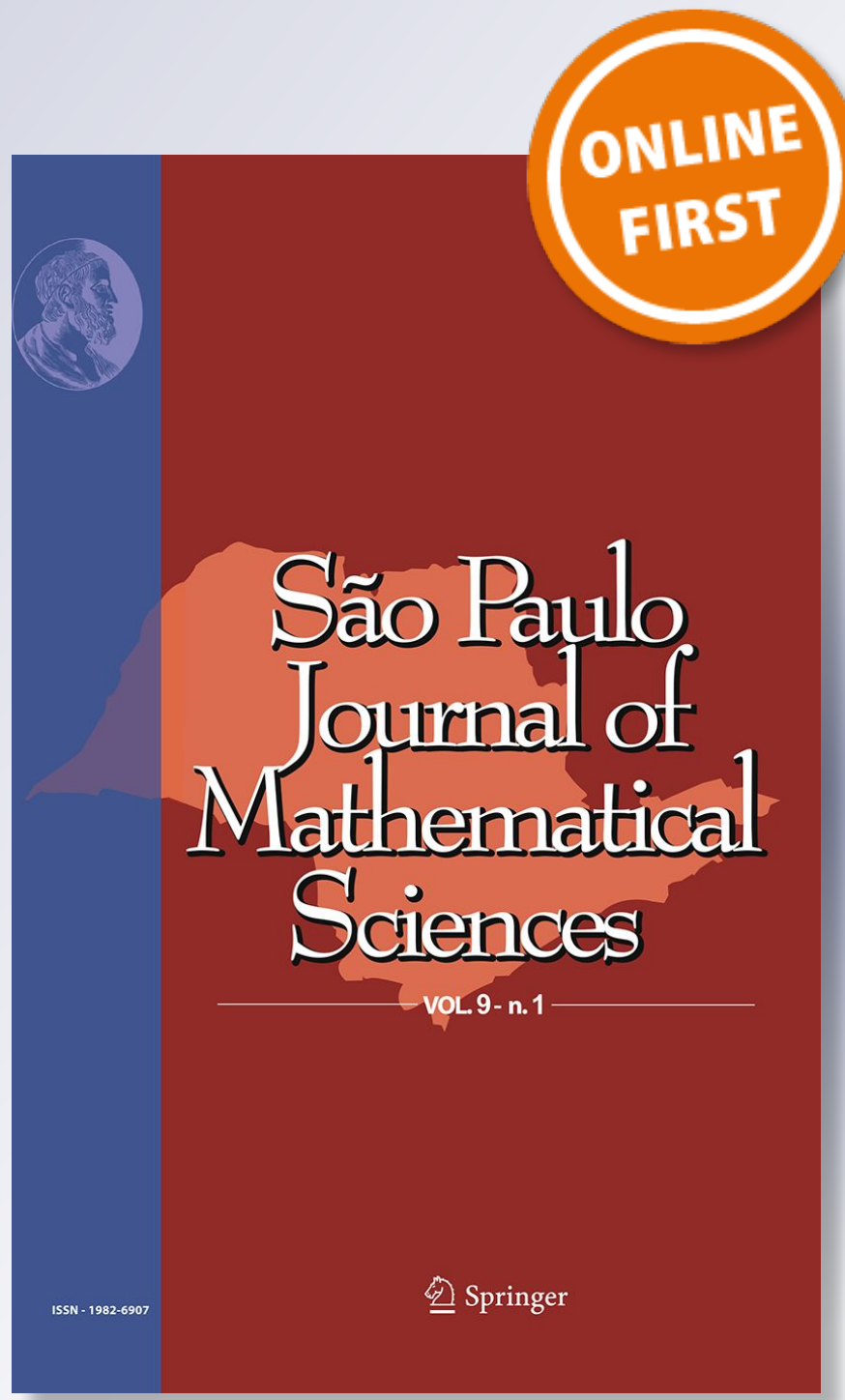

Springer 
Your article is protected by copyright and all rights are held exclusively by Instituto de Matemática e Estatística da Universidade de São Paulo. This e-offprint is for personal use only and shall not be self-archived in electronic repositories. If you wish to self-archive your article, please use the accepted manuscript version for posting on your own website. You may further deposit the accepted manuscript version in any repository, provided it is only made publicly available 12 months after official publication or later and provided acknowledgement is given to the original source of publication and a link is inserted to the published article on Springer's website. The link must be accompanied by the following text: "The final publication is available at link.springer.com". 


\title{
Brackets, superalgebras and spectral gap
}

\author{
Consuelo Martínez ${ }^{1}$ Efim Zelmanov ${ }^{2}$
}

C Instituto de Matemática e Estatística da Universidade de São Paulo 2019

\begin{abstract}
The purpose of this survey is to discuss Poisson and contact brackets and related infinite dimensional superalgebras. All vector spaces are considered over the field of complex numbers $\mathbb{C}$.
\end{abstract}

Keywords Bracket $\cdot$ Superalgebra $\cdot$ Conformal algebra

Mathematics Subject Classification 17B68 $\cdot 17$ B63 $\cdot 17$ C90

\section{Brackets}

Let $A$ be an associative commutative algebra. A binary bilinear operation [, ] : $A \times A \rightarrow A$ is called a Poisson bracket if

(1) $(A,[]$,$) is a Lie algebra,$

(2) $[a b, c]=a[b, c]+[a, c] b$ for arbitrary elements $a, b, c \in A$.

Example 1 Let $A$ be a polynomial algebra in $2 n$ variables $A=\mathbb{C}\left[p_{1}, \ldots, p_{n}\right.$, $\left.q_{1}, \ldots, q_{n}\right]$. Then

To our friend and colleague Ivan Shestakov with respect and affection.

The survey is based on our forthcoming paper with O. Mathieu. The authors thank him for useful discussions during the preparation of the survey.

$凶$ Consuelo Martínez

cmartinez@uniovi.es

Efim Zelmanov

ezelmano@math.ucsd.edu

1 Departmento de Matemáticas, Universidad de Oviedo, C/Calvo Sotelo, s/n, 33007 Oviedo, Spain

2 Department of Mathematics, University of California San Diego, 9500 Gilman Drive, La Jolla, CA 92093-0112, USA 


$$
[f, g]=\sum_{i=1}^{n}\left(\frac{\partial f}{\partial p_{i}} \frac{\partial g}{\partial q_{i}}-\frac{\partial g}{\partial p_{i}} \frac{\partial f}{\partial q_{i}}\right)
$$

is a Poisson bracket.

Example 2 Let $\mathfrak{g}$ be a Lie algebra. Let $A=\operatorname{Sym}(\mathfrak{g})$ be the symmetric tensor algebra over $\mathfrak{g}$. Then the Lie bracket on $\mathfrak{g}$ extends to a Poisson bracket on $A$.

A binary bilinear product $[]:, A \times A \rightarrow A$ is called a contact bracket if

(3) $(A,[]$,$) is a Lie algebra,$

(4) the linear transformation $D: a \mapsto[a, 1], a \in A$, is a derivation of the algebra $A$,

(5) $[a b, c]=a[b, c]+[a, c] b+a b D(c)$ for arbitrary elements $a, b, c \in A$.

Example 3 Every Poisson bracket is a contact bracket with derivation $D=0$.

Example 4 Let $A$ be an associative commutative algebra with a derivation $D$. Then $[a, b]=D(a) b-a D(b)$ is a contact bracket (of vector field type).

Example 5 Let $A=\mathbb{C}\left[p_{1}, \ldots, p_{n}, q_{1}, \ldots, q_{n}, t\right]$ be a polynomial algebra in $2 n+1$ variables. Let $D=\frac{\partial}{\partial t}$. In view of the formula (5) a contact bracket is uniquely determined by its values on generators (including 1). However, not all values on generators extend to a contact bracket. The values

$$
\left[p_{i}, q_{j}\right]=\delta_{i j},\left[t, p_{i}\right]=\frac{1}{2} D(t) p_{i},\left[t, q_{i}\right]=\frac{1}{2} D(t) q_{i}
$$

extend to a contact bracket on $A$.

Question 1 Let $\left(A,[,]_{1}\right),\left(B,[,]_{2}\right)$ be Poisson and contact brackets on algebras $A$, $B$ respectively. Is there always a way to define a contact bracket on $A \otimes B$ extending $[,]_{1}$ and $[,]_{2}$ ?

Examples 1 and 5 lead to the infinite dimensional Lie algebras $H(2 n)$ and $K(2 n+1)$ of Hamiltonian and contact types respectively. They should be viewed in the context of the family of Lie algebras of Cartan type together with $W(n)=\operatorname{Der} \mathbb{C}\left[t_{1}, \ldots, t_{n}\right]$ and

$$
S(n)=\left\{d=\sum_{i=1}^{n} f_{i}\left(t_{1}, \ldots, t_{n}\right) \frac{\partial}{\partial t_{i}} \in W(n) \mid \operatorname{div} d=\sum_{i=1}^{n} \frac{\partial f_{i}}{\partial t_{i}}=0\right\} .
$$

The Lie algebras $W(n), S(n), H(2 n), K(2 n+1)$ are simple and finitely presented (see [9]).

Question 2 Find reasonable conditions on a Poisson (resp. contact) bracket [, ] for the Lie algebra $L=(A,[]$,$) or [L, L]$ to be (i) finitely generated, (ii) finitely presented.

Question 3 To which extent does the Lie algebra $L=(A,[]$,$) determine the bracket?$ More precisely, is an isomorphism of the Lie algebras $\left(A,[,]_{1}\right) \rightarrow\left(B,[,]_{2}\right)$ always extendable to an isomorphism of brackets in some sense? 


\section{Superalgebras}

By a superalgebra we mean an algebra $A=A_{\overline{0}}+A_{\overline{1}}$ graded by the cyclic group $\mathbb{Z} / 2 \mathbb{Z}$ of order 2 .

Example 6 Let $m, n \geq 1$. Then the decomposition

$$
M_{m+n}(\mathbb{C})=\left(\frac{* \mid 0}{0 \mid *}\right)_{n}^{m}+\left(\frac{0 \mid *}{* \mid 0}\right)
$$

defines the structure of a superalgebra on the algebra of $(m+n) \times(m+n)$ matrices.

Example 7 Grassmann (exterior) algebra. Consider the algebra $G(n)$ presented by generators and relations

$$
G(n)=\left\langle 1, \xi_{1}, \ldots, \xi_{n} \mid \xi_{i} \xi_{j}+\xi_{j} \xi_{i}=0, \xi_{i}^{2}=0\right\rangle
$$

Then the set of ordered products $1, \xi_{i_{1}} \cdots \xi_{i_{k}}, i_{1}<\cdots<i_{k}$, is a basis of $G(n)$. Let

$$
\begin{aligned}
& G_{\overline{0}}(n)=\operatorname{span}\left(1, \xi_{i_{1}} \cdots \xi_{i_{k}}, k \text { is even }\right), \\
& G_{\overline{1}}(n)=\operatorname{span}\left(\xi_{i_{1}} \cdots \xi_{i_{k}}, k \text { is odd }\right),
\end{aligned}
$$

$G(n)=G_{\overline{0}}(n)+G_{\overline{1}}(n)$ is a superalgebra. Let $G$ be the union of the ascending chain $G(1) \subset G(2) \subset \ldots$ Clearly, $G=G_{\overline{0}}+G_{\overline{1}}$, where

$$
G_{\overline{0}}=\bigcup_{n \geq 1} G_{\overline{0}}(n), \quad G_{\overline{1}}=\bigcup_{n \geq 1} G_{\overline{1}}(n) .
$$

Definition For a superalgebra $A=A_{\overline{0}}+A_{\overline{1}}$ the subalgebra

$$
G(A)=A_{\overline{0}} \otimes G_{\overline{0}}+A_{\overline{1}} \otimes G_{\overline{1}}<A \otimes G
$$

is called the Grassmann envelope of $A$.

Let $\mathcal{V}$ be a variety of algebras, i.e. a class of algebras defined by identities.

Definition We say that a superalgebra $A$ is a $\mathcal{V}$-superalgebra if $G(A) \in \mathcal{V}$.

Considering the varieties of associative commutative, Lie, Jordan algebras we get classes of associative commutative, Lie, Jordan superalgebras.

These classes can be defined by graded identities. If the variety $\mathcal{V}$ is defined by multilinear identities and in some term of one of these identities variables $x_{i}, x_{j}$ are permuted then this term has to be multiplied by $(-1)^{\left|x_{i}\right| \cdot\left|x_{j}\right|}$, where $\left|x_{i}\right|,\left|x_{j}\right|$ are the parities of the variables $x_{i}, x_{j}$.

Example 8 The Grassmann algebra $G=G_{\overline{0}}+G_{\overline{1}}$ is an associative commutative superalgebra. 
Example 9 If $A \in \mathcal{V}$ then $A \otimes G=A \otimes G_{\overline{0}}+A \otimes G_{\overline{1}}$ is a $\mathcal{V}$-superalgebra.

The notions of Poisson and contact brackets can be easily "superized" in accordance with the above rule.

Definition Let $A=A_{\overline{0}}+A_{\overline{1}}$ be an associative commutative superalgebra. A graded binary bilinear product $[]:, A \times A \rightarrow A$ is called a Poisson bracket if

(6) $(A,[]$,$) is a Lie superalgebra,$

(7) $[a b, c]=a[b, c]+(-1)^{|b| \cdot|c|}[a, c] b$ for arbitrary elements $a, b, c \in A_{\overline{0}} \cup A_{\overline{1}}$.

Definition A graded binary bilinear product [, ] : $A \times A \rightarrow A$ is called a contact bracket if

(8) $(A,[]$,$) is a Lie superalgebra,$

(9) the linear transformation $D: a \mapsto[a, 1], a \in A$, is an even derivation of $A$,

(10) $[a b, c]=a[b, c]+(-1)^{|b| \cdot|c|}[a, c] b+a b D(c)$ for arbitrary elements $a, b, c \in$ $A_{\overline{0}} \cup A_{\overline{1}}$.

Example 10 The bracket

$$
[f, g]=\sum_{i=1}^{n}(-1)^{|f|} \frac{\partial f}{\partial \xi_{i}} \frac{\partial g}{\partial \xi_{i}}
$$

is a Poisson bracket on the Grassmann superalgebra $G$.

Let $\mathbb{C}\left[t_{1}, t_{1}^{-1}, \ldots, t_{m}, t_{m}^{-1}\right]$ be the algebra of Laurent polynomials in $m$ variables. Consider the associative commutative superalgebra

$$
\begin{aligned}
\Lambda(m: n) & =\mathbb{C}\left[t_{1}, t_{1}^{-1}, \ldots, t_{m}, t_{m}^{-1}\right] \otimes G(n)=\Lambda(m: n)_{\overline{0}}+\Lambda(m: n)_{\overline{1}} \\
\Lambda(m: n)_{\bar{i}} & =\mathbb{C}\left[t_{1}, t_{1}^{-1}, \ldots, t_{m}, t_{m}^{-1}\right] \otimes G(n)_{\bar{i}}, \quad i=0 \text { or } 1 .
\end{aligned}
$$

Example 11 Let $m=2 k$,

$$
\Lambda(m: n)=\mathbb{C}\left[p_{1}, p_{1}^{-1}, \ldots, p_{k}, p_{k}^{-1}, q_{1}, q_{1}^{-1}, \ldots, q_{k}, q_{k}^{-1}, \xi_{1}, \ldots, \xi_{n}\right] .
$$

Then

$$
[f, g]=\sum_{i=1}^{k}\left(\frac{\partial f}{\partial p_{i}} \frac{\partial g}{\partial q_{i}}-\frac{\partial g}{\partial p_{i}} \frac{\partial f}{\partial q_{i}}\right)+\sum_{i=1}^{n}(-1)^{|f|} \frac{\partial f}{\partial \xi_{i}} \frac{\partial g}{\partial \xi_{i}}
$$

is a Poisson bracket. It gives rise to the Hamiltonian Lie superalgebra $H(m: n)=$ $(\Lambda(m: n),[]$,$) .$

Example 12 Let $m=2 k+1$,

$$
\Lambda(m: n)=\mathbb{C}\left[p_{1}, p_{1}^{-1}, \ldots, p_{k}, p_{k}^{-1}, q_{1}, q_{1}^{-1}, \ldots, q_{k}, q_{k}^{-1}, t, t^{-1}, \xi_{1}, \ldots, \xi_{n}\right] .
$$


Let

$$
D= \begin{cases}\frac{\partial}{\partial t} & \text { Neveu-Schwarz type } \\ t \frac{\partial}{\partial t} & \text { Ramond type. }\end{cases}
$$

Let $\left[p_{i}, p_{j}\right]=\left[q_{i}, q_{j}\right]=0,\left[p_{i}, q_{j}\right]=\delta_{i j},\left[t, p_{i}\right]=\frac{1}{2} D(t) p_{i},\left[t, q_{i}\right]=$ $\frac{1}{2} D(t) q_{i},\left[t, \xi_{\ell}\right]=\frac{1}{2} D(t) \xi_{\ell},\left[p_{i}, \xi_{\ell}\right]=\left[q_{i}, \xi_{\ell}\right]=0,\left[\xi_{\ell}, \xi_{s}\right]=\delta_{\ell, s}$ for all $1 \leq i, j \leq k ; 1 \leq \ell, s \leq n$. These values extend to a contact bracket on $\Lambda(m: n)$. The bracket gives rise to the Lie superalgebra $K(m: n)=(\Lambda(m: n),[]$,$) .$

Along with superalgebras $H(m: n)$ and $K(m: n)$ we can consider other superalgebras of Cartan type. Denote

$$
W(m: n)=\operatorname{Der} \Lambda(m: n)=\left\{\sum_{i=1}^{m} f_{i} \frac{\partial}{\partial t_{i}}+\sum_{j=1}^{n} g_{j} \frac{\partial}{\partial \xi_{j}}\right\}
$$

For an $m$-tuple $\alpha=\left(\alpha_{1}, \ldots, \alpha_{m}\right), \alpha_{i} \in \mathbb{C}$ and $t^{\alpha}=t_{1}^{\alpha_{1}} \ldots t_{m}^{\alpha_{m}}$ consider the subsuperalgebra $S(m: n, \alpha)=$

$$
\left\{D \in W(m: n) \mid \operatorname{div}\left(t^{\alpha} D\right)=\sum_{i=1}^{m} \frac{\partial\left(t^{\alpha} f_{i}\right)}{\partial t_{i}}+\sum_{j=1}^{n}(-1)^{\left|g_{j}\right|} \frac{\partial\left(t^{\alpha} g_{j}\right)}{\partial \xi_{j}}=0\right\}
$$

of $W(m: n)$.

Moreover, some even variables $t_{i}$ may appear without their inverses. Denote

$$
\begin{aligned}
\Lambda\left(m_{1}: m_{2}: n\right)= & \mathbb{C}\left[t_{i}, t_{i}^{-1} \mid 1 \leq i \leq m_{1}\right] \\
& \otimes \mathbb{C}\left[t_{j} \mid m_{1}+1 \leq j \leq m_{1}+m_{2}\right] \otimes G(n) ; \\
W\left(m_{1}: m_{2}: n\right)= & \operatorname{Der} \Lambda\left(m_{1}: m_{2}: n\right) ; \\
S\left(m_{1}: m_{2}: n, \alpha\right)= & \left\{D \in W\left(m_{1}: m_{2}: n\right) \mid \operatorname{div}\left(t^{\alpha} D\right)=0\right\} .
\end{aligned}
$$

The superalgebras $H\left(m_{1}: m_{2}: n\right)$ and $K\left(m_{1}: m_{2}: n\right)$ can be viewed as subsuperalgebras of $H\left(m_{1}+m_{2}: n\right)$ and $K\left(m_{1}+m_{2}: n\right)$ respectively whose underlying subspace is $\Lambda\left(m_{1}: m_{2}: n\right)$.

Let us discuss connections between brackets and Jordan superalgebras.

Kantor [14] noticed that if $A$ is an associative commutative superalgebra with a Poisson bracket [, ] then the vector space $J=A+A v$ with an operation that extends the operation on $A$ and $a(b v)=a b v,(b v) a=(-1)^{|a|}(b a) v,(a v)(b v)=$ $(-1)^{|b|}[a, b] ; a, b \in A_{\overline{0}} \cup A_{\overline{1}}$ is a Jordan superalgebra. $J_{\overline{0}}=A_{\overline{0}}+A_{\overline{1}} v, J_{\overline{1}}=$ $A_{\overline{1}}+A_{\overline{0}} v, J=J_{\overline{0}}+J_{\overline{1}}$. We call it the Kantor double of the bracket [, ] and denote it by $K(A,[]$,$) . There exist, however, non-Poisson brackets whose Kantor$ doubles are Jordan superalgebras. We call such brackets Jordan brackets. Cantarini and Kac [4] noticed that Jordan brackets are in 1-1 correspondence with contact 
brackets. More precisely, if $[a, b]$ is a contact bracket with derivation $D(a)=[a, 1]$ then $\langle a, b\rangle=[a, b]-\frac{1}{2}(D(a) b-a D(b))$ is a Jordan bracket.

Tits [28,29] made the following observation. Let $L$ be a Lie (super)algebra, $L_{\overline{0}}$ contains $\mathfrak{s l}_{2}=\mathbb{C} e+\mathbb{C} f+\mathbb{C} h,[e, f]=h,[h, e]=2 e,[h, f]=-2 f$ (we call such triple $e, f, h$ an $\mathfrak{s l}_{2}$-triple). Suppose that the operator $\operatorname{ad}(h): L \rightarrow L$ is diagonalizable and has eigenvalues $-2,0,2$, so $L=L_{-2}+L_{0}+L_{2}$ is a direct sum of eigenspaces. Then $J=\left(L_{2}, a \cdot b=[[a, f], b]\right)$ is a Jordan (super)algebra.

Moreover, Tits [28,29], Kantor [13] and Koecher [16] showed that every Jordan (super)algebra can be obtained in this way. The corresponding Lie (super)algebra $L$ is not unique, but any two such Lie superalgebras are centrally isogenous. Let $L=\operatorname{TKK}(J)$ be the universal Lie (super)algebra in this class (see [19]).

Let [, ] be the Poisson bracket of Example 11 on $\Lambda(m: n)$, if $m$ is even, or corresponds to the contact bracket of Example 12 on $\Lambda(m: n)$, if $m$ is odd. Consider the Kantor double $J(m: n)=K(\Lambda(m: n),[]$,$) . Then$

$$
\operatorname{TKK}(J(m: n)) \cong K(m: n+3)
$$

\section{Superconformal algebras}

\subsection{Basic examples}

The centerless Virasoro algebra is the algebra of derivations of Laurent polynomials,

$$
\operatorname{Vir}=\operatorname{Der} \mathbb{C}\left[t, t^{-1}\right]=\left\{f(t) \frac{\partial}{\partial t}\right\}
$$

In view of importance of the Virasoro algebra and (especially) its central extensions in Physics Neveu, Schwarz [24], Ramond [25] and others considered superextensions of the algebra Vir. These superextensions became known as superconformal algebras. In [11] Kac and van de Leur put the theory on a more formal footing and recognized that all known superconformal algebras are in fact infinite dimensional superalgebras of Cartan type considered in [9]. Following [11] we call a $\mathbb{Z}$-graded Lie superalgebra $L=\sum_{i \in \mathbb{Z}} L_{i}$ a superconformal algebra if

(i) $L$ is graded simple,

(ii) Vir $\subseteq L_{\overline{0}}$,

(iii) the dimensions $\operatorname{dim} L_{i}, i \in \mathbb{Z}$, are uniformly bounded.

Example 13 The superalgebra $W(1: n)=\operatorname{Der} \mathbb{C}\left[t, t^{-1}, \xi_{1}, \ldots, \xi_{n}\right]$ graded by degrees in $t$ is a superconformal algebra.

Example 14 Let $\alpha \in \mathbb{C}$,

$$
S(n, \alpha)=\left\{D \in W(1: n) \mid \operatorname{div}\left(t^{\alpha} D\right)=0\right\}<W(1: n) .
$$


The superalgebra $S(n, \alpha)$ is simple unless $\alpha \in \mathbb{Z}$. If $\alpha \in \mathbb{Z}$ then $[S(n, \alpha), S(n, \alpha)]$ is a proper ideal in $S(n, \alpha)$ and the superalgebra $[S(n, \alpha], S(n, \alpha)]$ is simple. In this case we will change the notation

$$
S(n, \alpha):=[S(n, \alpha), S(n, \alpha)]
$$

Also $S(n, \alpha) \cong S(n, \beta)$ if and only if $\alpha-\beta \in \mathbb{Z}$, so this family of superalgebras is parametrized by $\alpha \in \mathbb{C} / \mathbb{Z}$. It appeared in Physics literature $[1,27]$ under the name "SU 2 -superconformal algebras".

Example 15 Consider the superalgebras $K(1: n)$. Abusing notation we will denote them as $K(n)$. The superalgebra $K(n)$ is simple except for $n=4$.

For $n=4$ the commutator ideal $[K(4), K(4)]$ has codimension 1 in $K(4)$ and $[K(4), K(4)]$ is a simple superalgebra. We will denote $K(4):=[K(4), K(4)]$.

The superalgebras $K(n)$ are $\mathbb{Z}$-graded. In the Ramond case they are graded by degrees in $t$. In the Neveu-Schwarz case we define $\operatorname{deg}\left(t^{i} \xi_{i_{1}} \cdots \xi_{i_{r}}\right)=2(i+1)-r$. We remark that this grading is not induced by a grading of an associative commutative superalgebra $\Lambda(1: n)$.

Thus, $K(n), n \geq 1$, is another family of superconformal algebras. In Physics literature it is known as the family of " $\mathrm{SO}_{n}$-superconformal algebras" [1,26]. The superalgebras $K(1)$ are just the Neveu-Schwarz and Ramond superalgebras. We remark that in the Neveu-Schwarz case the embedding Vir $\hookrightarrow K(n)_{\overline{0}}$ is not graded as $\operatorname{Vir}_{i} \hookrightarrow K(n)_{\overline{0}, 2 i}$.

Along with superalgebras $K(n)$ we consider their twisted versions. Let $\xi_{1}, \ldots, \xi_{n}$ be the Grassmann variables involved, $\left[\xi_{i}, \xi_{j}\right]=\delta_{i, j}$. The algebra $\Lambda(1: n)=$ $\mathbb{C}\left[t, t^{-1}, \xi_{1}, \ldots, \xi_{n}\right]$ is $\mathbb{Z} / 2 \mathbb{Z}$-graded: $\Lambda(1: n)=\mathbb{C}\left[t, t^{-1}, \xi_{1}, \ldots, \xi_{n-1}\right]+$ $\mathbb{C}\left[t, t^{-1}, \xi_{1}, \ldots, \xi_{n-1}\right] \xi_{n}=\Lambda(1: n-1)+\Lambda(1: n-1) \xi_{n}$.

Consider the (twisted) algebra $K(n, 1)=\Lambda(1: n-1)+\Lambda(1: n-1) t^{\frac{1}{2}} \xi_{n}$ with the bracket on it. For even $n$ the superalgebra $K(n, 1)$ is not isomorphic to any of the superalgebras $K(n)$ considered above.

Example 16 In 1996 Cheng-Kac [5] and, independently, Grozman-Leites-Shchepochkina [8] introduced a new family of superconformal algebras. Following [5] we will denote it as $\mathrm{CK}(6)$. In [22] for an arbitrary associative commutative superalgebra $R$ and an even derivation $D$ of $R$ we constructed a superalgebra $\operatorname{CK}(R, d)$ such that $\mathrm{CK}\left(\mathbb{C}\left[t, t^{-1}\right], \frac{\partial}{\partial t}\right)=\mathrm{CK}(6)$. Let us briefly recall the construction.

The simple finite dimensional Lie superalgebra $P(n-1)$ is the superalgebra of $2 n \times 2 n$ matrices of the type $\left(\begin{array}{cc}a & k \\ h & -a^{T}\end{array}\right)$, where $a, k, h$ are $n \times n$ matrices over $\mathbb{C}$, $\operatorname{tr}(a)=0, k^{T}=-k$ and $h^{T}=h$. The superalgebras $P(n)$ (the so-called "strange" series), $n \geq 3$ are centrally closed. However, $P(3)$ has a nontrivial central cover $\widehat{P(3)}$. Its existence follows from the fact that the Lie algebra skew $4(\mathbb{C})$ of skewsymmetric $4 \times 4$ matrices is a direct sum of two ideals skew $4=\mathfrak{s l}_{2}(\mathbb{C}) \oplus \mathfrak{s l}_{2}(\mathbb{C})$. For an arbitrary element $k \in \operatorname{skew}_{4}(\mathbb{C})$ we consider its decomposition $k=k^{\prime}+k^{\prime \prime}$ and let $\varphi(k)=k^{\prime}-k^{\prime \prime}$. The universal central cover $\widehat{P(3)}$ of $P(3)$ can be realized as the superalgebra of $8 \times 8$ matrices over the polynomial algebra $\mathbb{C}[d]$ of the type 


$$
\left(\begin{array}{cc}
a & k \\
\varphi(k) d+h & -a^{T}
\end{array}\right)+\alpha d \cdot I_{8}
$$

where $a, k, h$ are $4 \times 4$ matrices over $\mathbb{C}, \operatorname{tr}(a)=0, k^{T}=-k, h^{T}=h, \alpha \in \mathbb{C}$ and $I_{8}$ is the identity matrix.

Consider the associative Weyl algebra $W=\sum_{i \geq 0} R d^{i}$, where the variable $d$ does not commute with a coefficient $a \in R$, but $d a=a \bar{d}+d(a)$.

The superalgebra $\mathrm{CK}(R, d)$ is a subsuperalgebra of $8 \times 8$ matrices over $W$ generated by $\widehat{P(3)}$ and by all matrices $\left(\begin{array}{cc}e_{i j}(a) & 0 \\ 0 & -e_{j i}(a)\end{array}\right)$, where $a \in R, 1 \leq i \neq j \leq$ $4, e_{i j}(a)$ is the $4 \times 4$ matrix having the element $a$ at the intersection of the $i$-th row and $j$-th column, and zeros everywhere else.

A superalgebra $\mathrm{CK}(R, d)$ contains an $\mathfrak{s l}_{2}$-triple $e, f, h$ such that $\operatorname{ad}(h)$ has eigenvalues $-2,0,2$. Hence $\mathrm{CK}(R, d)$ is isomorphic to the Tits-Kantor-Koecher construction of a certain Jordan superalgebra that we will denote as $\operatorname{JCK}(R, d)$,

$$
\mathrm{CK}(R, d) \cong \operatorname{TKK}(\operatorname{JCK}(R, d)) .
$$

Conjecture (V. Kac-J. van de Leur) An arbitrary superconformal algebra is isomorphic to one of the superalgebras $W(1: n), S(n, \alpha), \alpha \in \mathbb{C} / \mathbb{Z}, K(n), K(n, 1), \mathrm{CK}(6)$.

In [10] we proved that if $J=\sum_{i \in \mathbb{Z}} J_{i}$ is a graded simple Jordan superalgebra having all dimensions $\operatorname{dim} J_{i}, i \in \mathbb{Z}$ uniformly bounded, then either $J$ has finitely many nonzero negative components or $J$ has finitely many nonzero positive components or $J$ is a (twisted) superalgebra $J(1: n)$ (see Sect.2) or $J$ is isomorphic to $\operatorname{JCK}\left(\mathbb{C}\left[t, t^{-1}\right], d\right)$ where $d=\frac{\partial}{\partial t}$ or $t \frac{\partial}{\partial t}$.

This theorem implies that the only superconformal algebras coming from Jordan superalgebras are $K(n), n \geq 3$, and $\mathrm{CK}(6)$ as envisioned by the Kac-van de Leur Conjecture.

In the next section we will consider another important case when the Conjecture has been proved.

\subsection{Polynomiality assumption}

Suppose that $L=\sum_{i \in \mathbb{Z}} L_{i}$ is a $\mathbb{Z}$-graded Lie superalgebra, dimensions $\operatorname{dim} L_{\overline{0} i}=$ $d_{0}, \operatorname{dim} L_{\overline{1} i}=d_{1}$ do not depend on $i, d=d_{0}+d_{1}$. Suppose further that there exist bases $e_{i 1}, \ldots, e_{i d}$ of $L_{i}$ such that all $d^{3}$ structural component functions

$$
\left[e_{i p}, e_{j q}\right]=\sum_{r=1}^{d} \gamma_{p q r}(i, j) e_{i+j, r}
$$

are polynomials in $i, j$.

Remark This assumption holds for all known superconformal algebras $W, S, K$, CK(6). 
We will recall some definitions and facts concerning formal distributions (for more details see [12]).

Let $A$ be an arbitrary (not necessarily associative) algebra. By a formal distribution

$$
a(z)=\sum_{i \in \mathbb{Z}} a(i) z^{-i-1} \in A\left[\left[z, z^{-1}\right]\right]
$$

we mean a power series over $A$ that is infinite in both directions.

Definition Two formal distributions $a(z), b(z)$ are said to be mutually local if there exists an integer $N=N(a, b) \geq 0$ such that

$$
a(z) b(w)(z-w)^{N}=b(w) a(z)(z-w)^{N}=0 .
$$

We will consider a countable family of operations

$$
a(z) \circ_{n} b(z)=\operatorname{Res}_{w} a(w) b(z)(w-z)^{n}, n \geq 0, n \in \mathbb{Z} .
$$

Here $\operatorname{Res}_{w}$ denotes the coefficient at $w^{-1}$.

If $a(z), b(z)$ are mutually local then only finitely many products $a \circ_{n} b$ may be different from zero.

Example 17 For $L$ one of the Lie superalgebras discussed above, the Polynomiality Assumption implies that the formal distributions $e_{p}(z)=\sum_{i \in \mathbb{Z}} e_{p i} z^{-i-1}, 1 \leq p \leq d$, are pairwise mutually local.

Definition A vector space $C \subseteq A\left[\left[z, z^{-1}\right]\right]$ is called a conformal algebra of formal distributions if $\partial C \subseteq C, \partial=\frac{d}{d z}, C \circ_{n} C \subseteq C$ for an arbitrary $n \geq 0$, and every two elements from $C$ are mutually local.

Dong Lemma (see [6,12]) Let A be an associative or Lie superalgebra and let distributions $a(z), b(z), c(z) \in A\left[\left[z, z^{-1}\right]\right]$ be pairwise mutually local. Then

(i) for an arbitrary $n \geq 0$ the distributions $a \circ_{n} b$ and $c$ are mutually local,

(ii) the distributions $\frac{d}{d z} a(z)$ and $b(z)$ are mutually local.

This lemma implies that if $A$ is an associative or Lie superalgebra, then an arbitrary family $\left\{a_{i}(z)\right\}$ of pairwise mutually local distributions over $A$ (we assume that each $a_{i}(z)$ is also mutually local with itself) generates a conformal algebra of formal distributions.

Example 18 In the superconformal algebras $W, S, K, \mathrm{CK}(6)$ the formal distributions

$$
e_{p}(z)=\sum_{i \in \mathbb{Z}} e_{p i} z^{-i-1}
$$

generate conformal algebras Conf $W(1: n)$, Conf $S(n, \alpha)$, Conf $K(n)$ and Conf CK(6). 
A conformal algebra is said to be of finite type if it is a finitely generated module over $\mathbb{C}[\partial]$. Fattori and Kac [7] proved that a simple Lie conformal superalgebra of finite type is isomorphic to a current superalgebra Conf $\mathfrak{g} \otimes \mathbb{C}\left[t, t^{-1}\right]$ or to one of the superalgebras Conf Vir, Conf $W(1: n)$, Conf $S(n, \alpha)$, Conf $K(n)$ or Conf CK(6). In the last two cases we include only the Neveu-Schwarz type. The conformal algebra that corresponds to the Ramond superalgebra is not of finite type.

This confirms the Kac-van de Leur Conjecture for superconformal algebras that correspond to conformal algebras of finite type.

\subsection{Cartan subalgebras and roots}

In each of the superalgebras $W, S, K, \mathrm{CK}(6)$ we will select an even toroidal subalgebra of zero degree and describe root decompositions with respect to it.

3.3.1 In $L=W(1: n)=\left\{f(t, \xi) \frac{\partial}{\partial t}+\sum_{i=1}^{n} f_{i}(t, \xi) \frac{\partial}{\partial \xi_{i}}\right\}$ consider the Cartan subalgebra $H=\left\{\sum_{i=1}^{n} \alpha_{i} \xi_{i} \frac{\partial}{\partial \xi_{i}} \mid \alpha_{i} \in \mathbb{C}\right\}$ and its subalgebra $H^{(0)}=\left\{\sum_{i=1}^{n} \alpha_{i} \xi_{i} \frac{\partial}{\partial \xi_{i}} \mid\right.$ $\left.\sum_{i=1}^{n} \alpha_{i}=0\right\}$. The subalgebra $H^{(0)}$ is spanned by elements $\xi_{i} \frac{\partial}{\partial \xi_{i}}-\xi_{j} \frac{\partial}{\partial \xi_{j}}$. We notice that for each $1 \leq i \neq j \leq n$ the elements $\xi_{i} \frac{\partial}{\partial \xi_{j}}, \xi_{i} \frac{\partial}{\partial \xi_{i}}-\xi_{j} \frac{\partial}{\partial \xi_{j}}, \xi_{j} \frac{\partial}{\partial \xi_{i}}$ form an $\mathfrak{s l}_{2}$-triple lying in $L_{\overline{0} 0}$.

Let $\omega_{i} \in H^{*}$ denote the functional $\left\langle\omega_{i}, \sum_{j=1}^{n} \alpha_{j} \xi_{j} \frac{\partial}{\partial \xi_{j}}\right\rangle=\alpha_{i}, 1 \leq i \leq n$. The action of $H$ on $L$ defines a decomposition of $L$ into the sum of the centralizer

$$
C_{L}(H)=\mathbb{C}\left[t, t^{-1}\right] \frac{\partial}{\partial t}+\sum_{i=1}^{n} \mathbb{C}\left[t, t^{-1}\right] \xi_{i} \frac{\partial}{\partial \xi_{i}}=\mathbb{C}\left[t, t^{-1}\right] \frac{\partial}{\partial t}+\mathbb{C}\left[t, t^{-1}\right] H,
$$

and a finite collection of eigenspaces

$$
L=C_{L}(H)=\sum_{0 \neq \alpha \in \Delta} L_{\alpha}
$$

$\Delta=\left\{\omega_{i_{1}}+\cdots+\omega_{i_{r}}, \omega_{i_{1}}+\cdots+\omega_{i_{r}}-\omega_{i}\right\} \subset \bigoplus_{i=1}^{n} \mathbb{Z} \omega_{i}$. Functionals $\alpha \in \Delta$ are called roots. For a $\operatorname{root} \alpha=\omega_{i_{1}}+\cdots+\omega_{i_{r}}, 1 \leq i_{1}<\cdots<i_{r} \leq n$ we have

$$
L_{\alpha}=\mathbb{C}\left[t, t^{-1}\right] \xi_{i_{1}} \ldots \xi_{i_{r}} \frac{\partial}{\partial t}+\mathbb{C}\left[t, t^{-1}\right] \xi_{i_{1}} \ldots \xi_{i_{r}} H
$$

For a root $\alpha=\omega_{i_{1}}+\cdots+\omega_{i_{r}}-\omega_{j}, j \notin\left\{1, \ldots, i_{r}\right\}, 1 \leq i_{1}<\cdots<i_{r} \leq n$, we have

$$
L_{\alpha}=\mathbb{C}\left[t, t^{-1}\right] \xi_{i_{1}} \ldots \xi_{i_{r}} \frac{\partial}{\partial \xi_{j}}
$$


Now consider a functional $f: \bigoplus_{i=1}^{n} \mathbb{C} \omega_{i} \rightarrow \mathbb{C}, f\left(\omega_{i}\right)=2^{i-1}, 1 \leq i \leq n$. Clearly, $f(\alpha) \neq 0$ for an arbitrary $\operatorname{root} \alpha \in \Delta$. Then $\Delta=\Delta_{-} \dot{\cup} \Delta_{+}$, where

$$
\begin{aligned}
\Delta_{-} & =\{\alpha \in \Delta \mid f(\alpha)<0\}=\left\{\omega_{i_{1}}+\cdots+\omega_{i_{r}}-\omega_{j}, i_{r}<j\right\} \\
\Delta_{+} & =\{\alpha \in \Delta \mid f(\alpha)>0\} \\
& =\left\{\omega_{i_{1}}+\cdots+\omega_{i_{r}}, \omega_{i_{1}}+\cdots+\omega_{i_{r}}-\omega_{j}, j<i_{r}\right\}
\end{aligned}
$$

Let

$$
L_{-}=\sum_{\alpha \in \Delta_{-}} L_{\alpha}, \quad L_{+}=\sum_{\alpha \in \Delta_{+}} L_{\alpha}, \quad B=C_{L}(H)+L_{+} .
$$

The subsuperalgebra $B$ is the Borel subalgebra of $L$.

3.3.2 Let $L=S(n, \gamma), \gamma \in \mathbb{C}$. For an arbitrary $k \in \mathbb{Z}$ the elements

$$
\begin{gathered}
e_{k}=-t^{k+1} \frac{\partial}{\partial t}+\frac{\gamma+k+1}{2} t^{k}\left(\xi_{1} \frac{\partial}{\partial \xi_{1}}+\xi_{2} \frac{\partial}{\partial \xi_{2}}\right) \quad \text { and } \\
t^{k} \xi_{i} \frac{\partial}{\partial \xi_{j}}, \quad t^{k}\left(\xi_{i} \frac{\partial}{\partial \xi_{i}}-\xi_{j} \frac{\partial}{\partial \xi_{j}}\right), 1 \leq i \neq j \leq n,
\end{gathered}
$$

lie in $S(n, \gamma)$. We have $\left[e_{i}, e_{j}\right]=(i-j) e_{i+j}$, hence $\sum_{i \in \mathbb{Z}} \mathbb{C} e_{i}=$ Vir.

The subalgebra $H^{(0)}=\left\{\sum_{i=1}^{n} \alpha_{i} \xi_{i} \frac{\partial}{\partial \xi_{i}} \mid \sum_{i=1}^{n} \alpha_{i}=0\right\}$ lies in $S(n, \gamma)$ and

$$
S(n, \gamma)=\sum_{\alpha \in \Delta \cup\{0\}} S(n, \gamma) \cap W(1: n)_{\alpha}, \Delta \subseteq \bigoplus_{i=1}^{n} \mathbb{Z} \omega_{i},
$$

which defines a $\bigoplus_{i=1}^{n} \mathbb{Z} \omega_{i}$-grading on $S(n, \gamma)$. The centralizer of $H^{(0)}$ in $S(n, \gamma)$ is $\operatorname{Vir}+\mathbb{C}\left[t, t^{-1}\right] H^{(0)}$.

Let

$$
\begin{aligned}
& S(n, \gamma)_{+}=\sum_{\alpha \in \Delta_{+}} S(n, \gamma) \cap W(1: n)_{\alpha}, \\
& S(n, \gamma)_{-}=\sum_{\alpha \in \Delta_{-}} S(n, \gamma) \cap W(1: n)_{\alpha} .
\end{aligned}
$$

Then

$$
S(n, \gamma)=S(n, \gamma)_{+}+\left(\operatorname{Vir}+\mathbb{C}\left[t, t^{-1}\right] H^{(0)}\right)+S(n, \gamma)_{-}
$$


3.3.3 Let $L=K(2 n), n \geq 1$. Up to a change of a basis we can assume that the Grassmann variables are $\xi_{1}, \ldots, \xi_{n}, \eta_{1}, \ldots, \eta_{n}$, such that $\left[\xi_{i}, \xi_{j}\right]=\left[\eta_{i}, \eta_{j}\right]=0$ and $\left[\xi_{i}, \eta_{j}\right]=\delta_{i j}, 1 \leq i, j \leq n$.

The Cartan subalgebra of $L$ is $H=\left\{\sum_{i=1}^{n} \alpha_{i} \xi_{i} \eta_{i} \mid \alpha_{i} \in \mathbb{C}\right\}$. The centralizer of $H$ in $L$ is

$$
C_{L}(H)=\mathbb{C}\left[t, t^{-1}\right]+\sum_{1 \leq i_{1}<\cdots<i_{r} \leq n} \mathbb{C}\left[t, t^{-1}\right] \xi_{i_{1}} \eta_{i_{1}} \ldots \xi_{i_{r}} \eta_{i_{r}}
$$

In the centralizer $C_{L}(H)$ consider the nilpotent ideal

$$
\operatorname{Rad} C_{L}(H)=\sum_{1 \leq i_{1}<\cdots<i_{r} \leq n, r \geq 2} \mathbb{C}\left[t, t^{-1}\right] \xi_{i_{1}} \eta_{i_{1}} \ldots \xi_{i_{r}} \eta_{i_{r}}
$$

Then $C_{L}(H)=\mathbb{C}\left[t, t^{-1}\right]+\mathbb{C}\left[t, t^{-1}\right] H+\operatorname{Rad} C_{L}(H)$. As above we consider functionals $\omega_{i} \in H^{*},\left\langle\omega_{i}, \xi_{j} \eta_{j}\right\rangle=\delta_{i j}$. The action of $H$ on $L$ defines the root decomposition

$$
\begin{aligned}
L= & C_{L}(H)+\sum_{\alpha \in \Delta} L_{\alpha}, \\
\Delta= & \left\{\omega_{i_{1}}+\cdots+\omega_{i_{p}}-\omega_{j_{1}}-\cdots-\omega_{j_{q}} ;\right. \\
& 1 \leq i_{1}<\cdots<i_{p} \leq n ; 1 \leq j_{1}<\cdots<j_{q} \leq n ; \\
& \left.\left\{i_{1}, \ldots, i_{p}\right\} \cap\left\{j_{1}, \ldots, j_{q}\right\}=\emptyset\right\}
\end{aligned}
$$

For $\alpha=\omega_{i_{1}}+\cdots+\omega_{i_{p}}-\omega_{j_{1}}-\cdots-\omega_{j_{q}}$ we have

$$
L_{\alpha}=C_{L}(H) \xi_{i_{1}} \ldots \xi_{i_{p}} \eta_{j_{1}} \ldots \eta_{j_{q}}
$$

As above, consider the functional $f: H^{*} \rightarrow \mathbb{C}, f\left(\omega_{i}\right)=2^{i-1}, i \leq i \leq n$. Then $f(\alpha) \neq 0$ for all $\alpha \in \Delta$ and therefore

$$
\begin{aligned}
& \Delta=\Delta_{+} \dot{\cup} \Delta_{-}, \Delta_{+}=\{\alpha \in \Delta \mid f(\alpha)>0\}, \Delta_{-}=\{\alpha \in \Delta \mid f(\alpha)<0\}, \\
& L=L_{-}+C_{L}(H)+L_{+}, L_{-}=\sum_{\alpha \in \Delta_{-}} L_{\alpha}, L_{+}=\sum_{\alpha \in \Delta_{+}} L_{\alpha},
\end{aligned}
$$

$B=C_{L}(H)+L_{+}$is the Borel subalgebra.

3.3.4 $L=K(2 n+1), n \geq 1$. Again, up to a change of basis we can assume that the Grassmann variables are $\xi_{1}, \ldots, \xi_{n}, \eta_{1}, \ldots, \eta_{n}, \mu$; the bracket on $\xi_{i}, \eta_{j}$ is the same as in the case $K(2 n),\left[\xi_{i}, \mu\right]=\left[\eta_{i}, \mu\right]=0$ and $[\mu, \mu]=1$.

The Cartan subalgebra is the same,

$$
H=\left\{\sum_{i=1}^{n} \alpha_{i} \xi_{i} \eta_{i} \mid \alpha_{i} \in \mathbb{C}\right\}
$$


but

$$
\begin{aligned}
C_{L}(H)= & \left(\mathbb{C}\left[t, t^{-1}\right]+\mathbb{C}\left[t, t^{-1}\right] \mu\right) \\
& +\sum_{1 \leq i<\cdots<i_{r} \leq n}\left(\mathbb{C}\left[t, t^{-1}\right]+\mathbb{C}\left[t, t^{-1}\right] \mu\right) \xi_{i_{1}} \eta_{i_{1}} \ldots \xi_{i_{r}} \eta_{i_{r}}
\end{aligned}
$$

As above

$$
\begin{aligned}
\operatorname{Rad} C_{L}(H) & =\sum_{1 \leq i_{1}<\cdots<i_{r} \leq n, r \geq 2}\left(\mathbb{C}\left[t, t^{-1}\right]+\mathbb{C}\left[t, t^{-1}\right] \mu\right) \xi_{i_{1}} \eta_{i_{1}} \ldots \xi_{i_{r}} \eta_{i_{r}}, \\
C_{L}(H) & =\mathbb{C}\left[t, t^{-1}, \mu\right]+\mathbb{C}\left[t, t^{-1}, \mu\right] H+\operatorname{Rad} C_{L}(H)
\end{aligned}
$$

We remark that $\mathbb{C}\left[t, t^{-1}, \mu\right]=K(1)$ is the Neveu-Schwarz or Ramond superalgebra.

The root system and the functional $f: H^{*} \rightarrow \mathbb{C}$ that defines the decomposition $\Delta=$ $\Delta_{-} \dot{\cup} \Delta_{+}$are the same as in the case of $K(2 n)$. For $\alpha=\omega_{i_{1}}+\cdots+\omega_{i_{p}}-\omega_{j_{1}}-\cdots-\omega_{j_{q}}$ we have $L_{\alpha}=C_{L}(H) \xi_{i_{1}} \ldots \xi_{i_{p}} \eta_{j_{1}} \ldots \eta_{j_{q}}$.

3.3.5 $L=\mathrm{CK}(6)$. Recall that the Lie superalgebra $\mathrm{CK}(R, d)$ has been realized as a subsuperalgebra of $8 \times 8$ matrices over the Weyl algebra $W=\sum_{i \geq 0} R d^{i}$. The Cartan subalgebra in this case is

$$
H=\left\{\operatorname{diag}\left(\alpha_{1}, \alpha_{2}, \alpha_{3}, \alpha_{4},-\alpha_{1},-\alpha_{2},-\alpha_{3},-\alpha_{4}\right) \mid \alpha_{i} \in \mathbb{C}, \sum_{i=1}^{4} \alpha_{i}=0\right\}
$$

As above, define functionals $\omega_{i} \in H^{*}$ via

$$
\left\langle\omega_{i}, \operatorname{diag}\left(\alpha_{1}, \ldots, \alpha_{4},-\alpha_{1}, \ldots,-\alpha_{4}\right)\right\rangle=\alpha_{i}, \quad 1 \leq i \leq 4
$$

The dual space $H^{*}$ can be identified with

$$
\sum_{i=1}^{4} \mathbb{C} \omega_{i} / \mathbb{C}\left(\omega_{1}+\omega_{2}+\omega_{3}+\omega_{4}\right)
$$

The coset of $\omega_{i}$ maps $h=\operatorname{diag}\left(\alpha_{1}, \ldots, \alpha_{4},-\alpha_{1}, \ldots,-\alpha_{4}\right)$ to $\alpha_{i}$ for $1 \leq i \leq 4$. Then the even roots are $\Delta_{\overline{0}}=\left\{\omega_{i}-\omega_{j} \mid 1 \leq i \neq j \leq 4\right\}$, and the odd roots are $\Delta_{\overline{1}}=\left\{\omega_{i}+\omega_{j} \mid 1 \leq i \neq j \leq 4\right\} \cup\left\{-\omega_{i}-\omega_{j} \mid 1 \leq i, j \leq 4\right\}, \Delta=\Delta_{\overline{0}} \cup \Delta_{\overline{1}}$.

Consider the following root elements of $\operatorname{CK}(R, d)$ :

$$
\begin{aligned}
e_{\omega_{i}-\omega_{j}} & =\left(\begin{array}{cc}
e_{i j} & 0 \\
0 & -e_{j i}
\end{array}\right), \quad e_{\omega_{i}-\omega_{j}}(a)=\left(\begin{array}{cc}
e_{i j}(a) & 0 \\
0 & -e_{j i}(a)
\end{array}\right), \\
h_{\omega_{i}-\omega_{j}} & =\left(\begin{array}{cc}
e_{i i}-e_{j j} & 0 \\
0 & e_{j j}-e_{i i}
\end{array}\right),
\end{aligned}
$$




$$
\begin{aligned}
h_{\omega_{i}-\omega_{j}}(a) & =\left(\begin{array}{cc}
e_{i i}(a)-e_{j j}(a) & 0 \\
0 & e_{j j}(a)-e_{i i}(a)
\end{array}\right), \\
q_{\omega_{i}+\omega_{j}} & =\left(\begin{array}{cc}
0 & e_{i j}-e_{j i} \\
\varphi\left(e_{i j}-e_{j i}\right) d & 0
\end{array}\right),
\end{aligned}
$$

where $a \in R$.

In [20] it was shown that

$$
\begin{aligned}
\mathrm{CK}(R, d)_{\omega_{i}-\omega_{j}} & =e_{\omega_{i}-\omega_{j}}(R), \quad 1 \leq i \neq j \leq 4 ; \\
\mathrm{CK}(R, d)_{-2 \omega_{i}}= & q_{-2 \omega_{i}}(R) ; \\
\mathrm{CK}(R, d)_{\omega_{i}+\omega_{j}}= & {\left[q_{\omega_{i}+\omega_{k}}, e_{\omega_{j}-\omega_{k}}(R)\right]+q_{-\omega_{k}-\omega_{l}}(R), } \\
& \text { where }\{i, j, k, l\}=\{1,2,3,4\} .
\end{aligned}
$$

For an arbitrary element $a \in R$ consider the element

$$
\begin{aligned}
\operatorname{Vir}(a) & =\left[\left[e_{\omega_{4}-\omega_{1}}(a), q_{\omega_{3}+\omega_{1}}\right], q_{\omega_{2}+\omega_{1}}\right] \\
& =I_{8}(a d)-\left(\begin{array}{cc}
e_{11}\left(a^{\prime}\right) & 0 \\
0 & -e_{11}\left(a^{\prime}\right)+I_{4}\left(a^{\prime}\right)
\end{array}\right),
\end{aligned}
$$

where $a^{\prime}=[a, d]=d(a), I_{n}(a)=\operatorname{diag}(\underbrace{a, \ldots, a}_{n})$.

The mapping $R d \rightarrow \operatorname{Vir}(R), a d \mapsto \operatorname{Vir}(a)$ is an isomorphism from $\operatorname{Vir}$ to $\operatorname{Vir}(R)$. In [20] it was shown that

$$
C_{\mathrm{CK}(R, d)}(H)=\sum_{1 \leq i \neq j \leq 4} h_{\omega_{i}-\omega_{j}}(R)+\operatorname{Vir}(R) \cong R \otimes H+\operatorname{Vir}(R) .
$$

Consider the functional

$$
f: \sum_{i=1}^{4} \mathbb{C} \omega_{i} / \mathbb{C}\left(\omega_{1}+\omega_{2}+\omega_{3}+\omega_{4}\right) \rightarrow \mathbb{C}
$$

given by $f\left(\omega_{1}\right)=5, f\left(\omega_{2}\right)=-3, f\left(\omega_{3}\right)=2, f\left(\omega_{4}\right)=-4$. Notice that $f\left( \pm \omega_{i} \pm\right.$ $\left.\omega_{j}\right) \neq 0$ unless $\pm \omega_{i} \pm \omega_{j}=0$. This defines the decomposition $\Delta=\Delta_{+} \dot{\cup} \Delta_{-}$, and the triangular decomposition

$$
\mathrm{CK}(R, d)=\mathrm{CK}(R, d)_{-}+C_{\mathrm{CK}(R, d)}(H)+\mathrm{CK}(R, d)_{+} .
$$

\section{Harish-Chandra modules and spectral gaps}

Let $L$ be a superconformal algebra. A $\mathbb{Z}$-graded module $V$ over $L$ is called a HarishChandra module if all homogeneous subspaces $V_{i}, i \in \mathbb{Z}$ are finite dimensional. It looks as a natural class of representations for superconformal algebras. 
Let $\widehat{\text { Vir }}$ be the central cover of the (centerless) Virasoro algebra Vir.

Mathieu [23] and Martin and Piard [21] (under an additional assumption) classified irreducible Harish-Chandra modules over $\widehat{\operatorname{Vir}}$. Let $V=\sum_{i \in \mathbb{Z}} V_{i}$ be an irreducible Harish-Chandra module over $\widehat{\operatorname{Vir}}$. Then $\left\{i>0 \mid V_{i} \neq(0)\right\}$ is finite or $\left\{i<0 \mid V_{i} \neq(0)\right\}$ is finite or $V$ is a so-called intermediate module (see [15]). Being intermediate means that as a graded vector space $V$ is identified with the space of Laurent polynomials, $V=\overline{\mathbb{C}\left[t, t^{-1}\right]}$ and there exist scalars $\alpha, \beta \in \mathbb{C}$ such that

$$
\left(f(t) \frac{d}{d t}\right) \overline{g(t)}=\overline{-f g^{\prime}+\alpha f^{\prime} g+\beta \frac{1}{t} f g} .
$$

Denote $V=\Omega(\alpha, \beta)$. These modules are irreducible unless $\alpha \in \mathbb{Z}$. For $\alpha \in \mathbb{Z}$ we add factors of these modules to the list of intermediate modules.

Let $L$ be a superconformal algebra and let $\hat{L}$ be its central cover (in [11] Kac and van de Leur described superconformal algebras $W, S, K$ of small ranks that have nontrivial central extensions).

Let $\mathcal{S}$ be the category of graded $\hat{L}$-modules $V=\sum_{i \in \mathbb{Z}} V_{i}$ such that

(i) the center of $\hat{L}$ acts trivially, so, in fact, we are talking about $L$-modules,

(ii) the dimensions $\operatorname{dim} V_{i}, i \in \mathbb{Z}$ are uniformly bounded.

As in the case of modules over the Virasoro algebra the following proposition holds. Proposition 1 Let $V$ be an irreducible Harish-Chandra module over $\hat{L}$. Then either the set $\left\{i>0 \mid V_{i} \neq(0)\right\}$ is finite or the set $\left\{i<0 \mid V_{i} \neq(0)\right\}$ is finite or $V$ belongs to the category $\mathcal{S}$.

From now on we will focus on irreducible modules from the category $\mathcal{S}$.

\subsection{Harish-Chandra modules over Neveu-Schwarz and Ramond superalgebras}

Let $A$ be an associative commutative superalgebra with a contact bracket $[],, L=$ $(A,[]$,$) . Let B$ be an associative commutative superalgebra that contains $A, v \in B_{\overline{0}}$. Suppose that the bracket [, ] extends to a bracket on $B$ and $[A, A v] \subseteq A v$. Then $A v$ is a module over the Lie algebra $L$. We call it a bracket module.

Intermediate modules $\Omega(\alpha, \beta)$ are bracket modules. Indeed, $\operatorname{Vir} \cong(A,[]$,$) ,$ $A=\mathbb{C}\left[t, t^{-1}\right]$,

$$
[f(t), g(t)]=f^{\prime}(t) g(t)-f(t) g^{\prime}(t) .
$$

Let $B=\mathbb{C}\left[t, t^{-1}, v \mid v^{2}=0\right]$. The bracket [, ] extends to $B$ via $[1, v]=\alpha+$ $\beta,[t, v]=2 \alpha+\beta$. Then the Vir-module $\mathbb{C}\left[t, t^{-1}\right] v$ is isomorphic to $\Omega(\alpha, \beta)$.

Now we will introduce intermediate modules over Neveu-Schwarz and Ramond superalgebras. These superalgebras are isomorphic to $(A,[]),, A=\mathbb{C}\left[t, t^{-1}, \xi\right]$. Consider the superalgebra $B=\mathbb{C}\left[t, t^{-1}, v, \xi \mid v^{2}=0\right]$, where $v$ is an even element of degree 0 . The bracket [, ] extends to $B$ via

$$
[1, v]=\alpha+\beta, \quad[t, v]=(2 \alpha+\beta) t v,[\xi, v]=\frac{1}{2}(\alpha+\beta) \xi v
$$


The $L$-module $A v$ is irreducible unless $\alpha \in \mathbb{Z}$. If $\alpha \in \mathbb{Z}$ then it has a one dimensional submodule whose factor module is irreducible. The modules $A v$ and their factors (when applicable) are referred to as intermediate modules.

Theorem 4.1 Let L be a Neveu-Schwarz or Ramond superalgebra. An irreducible $L$-module from the category $\mathcal{S}$ is intermediate.

\subsection{Highest weight modules}

Since irreducible modules $V \in \mathcal{S}$ over superconformal algebras $W, S, K, \mathrm{CK}(6)$ of higher ranks are induced from modules over $C_{L}(H)$ we will start with irreducible modules over $C_{L}(H)$.

Let $\mathfrak{g}$ be the Virasoro algebra (resp. Neveu-Schwarz or Ramond superalgebra). Let $H$ be a finite dimensional vector space. Since $\mathfrak{g}$ naturally acts on $\mathbb{C}\left[t, t^{-1}\right]$ (resp. $\left.\mathbb{C}\left[t, t^{-1}, \mu\right]\right)$ it follows that $\mathbb{C}\left[t, t^{-1}\right] \otimes H\left(\right.$ resp. $\left.\mathbb{C}\left[t, t^{-1}, \mu\right] \otimes H\right)$ is a module over $\mathfrak{g}$. Let $L=\mathfrak{g}+M$ be the split extension, i.e. $[M, M]=(0)$.

Theorem 4.2 Let $V \in \mathcal{S}$ be a graded irreducible L-module. Then either $M V=(0)$ or $V=\overline{\mathbb{C}\left[t, t^{-1}\right]}$ (resp. $\overline{\mathbb{C}\left[t, t^{-1}, \mu\right]}$ ) and there exists a nonzero functional $\lambda \in H^{*}$ such that

$$
\begin{aligned}
(f(t) \otimes h) \overline{p(t)} & =\langle\lambda \mid h\rangle \overline{f(t) p(t)}, \\
((f(t, \mu) \otimes h) \overline{p(t, \mu)} & =\langle\lambda \mid h\rangle \overline{f(t, \mu) p(t, \mu)}, \text { respectively }) .
\end{aligned}
$$

If $\lambda=0$ then the module above is an intermediate $\mathfrak{g}$-module that corresponds to some scalars $\alpha, \beta \in \mathbb{C}$. If $\lambda \neq 0$ then the module is $\Omega(\alpha, \beta)$ for some $\alpha, \beta \in \mathbb{C}$.

In any case we will denote it as $\operatorname{Irr}(\lambda \mid \alpha, \beta)$.

The subspace $M$ acts on $\operatorname{Irr}(\lambda \mid \alpha, \beta)$ as described above.

Now let $L$ be one of the superalgebras

$$
W(1: n), n \geq 2 ; \quad S(n, \alpha), n \geq 2 ; \quad K(n), n \geq 2 ; \quad \mathrm{CK}(6)
$$

For each of them we have the decomposition $L=L_{-}+C_{L}(H)+L_{+}$, where $H$ is the Cartan subalgebra.

In the cases $W, S, \mathrm{CK}(6)$ we have $C_{L}(H) \cong \operatorname{Vir}+\mathbb{C}\left[t, t^{-1}\right] \otimes H$. In the case $K(2 n)$ we have $C_{L}(H) / \operatorname{Rad} C_{L}(H) \cong \operatorname{Vir}+\mathbb{C}\left[t, t^{-1}\right] \otimes H$ and, finally, in the case $K(2 n+1)$ we have $C_{L}(H) / \operatorname{Rad} C_{L}(H) \cong \mathfrak{g}+\mathbb{C}\left[t, t^{-1}, \mu\right] \otimes H$, where $\mathfrak{g}$ is the Neveu-Schwarz or Ramond superalgebra.

In all cases it allows us to view $\operatorname{Irr}(\lambda \mid \alpha, \beta)$ as an irreducible $C_{L}(H)$-module (in the case $K$ we assume $\left.\operatorname{Rad} C_{L}(H) \operatorname{Irr}(\lambda \mid \alpha, \beta)=(0)\right)$.

Let $\tilde{V}(\lambda \mid \alpha, \beta)$ be the universal "Verma type" $L$-module generated by $\operatorname{Irr}(\lambda \mid \alpha, \beta)$ and defined by relations:

(i) $L_{+} \operatorname{Irr}(\lambda \mid \alpha, \beta)=(0)$,

(ii) $C_{L}(H)$ acts on $\operatorname{Irr}(\lambda \mid \alpha, \beta)$ as described above. 
The module $\tilde{V}(\lambda \mid \alpha, \beta)$ has a largest submodule $M(\lambda \mid \alpha, \beta)$ such that $M(\lambda \mid$ $\alpha, \beta) \cap \operatorname{Irr}(\lambda \mid \alpha, \beta)=(0)$. Consider the irreducible $L$-module

$$
V(\lambda \mid \alpha, \beta)=\tilde{V}(\lambda \mid \alpha, \beta) / M(\lambda \mid \alpha, \beta)
$$

over $L$.

Let $V$ be a graded irreducible $L$-module from the category $\mathcal{S}$. The action of the Cartan subalgebra $H$ on $V$ is diagonalizable. For a functional $v \in H^{*}$ denote

$$
V_{v}=\{v \in V \mid h v=\langle v \mid h\rangle v \text { for all } h \in H\}
$$

The set of weights $\left\{v \in H^{*} \mid V_{v} \neq(0)\right\}$ is finite. In the cases $S, K, \mathrm{CK}(6)$ it follows from representation theory of $\mathfrak{s l}_{2}(\mathbb{C})$. In the case $W(1: n)$ one has to verify that the action of the element $\sum_{i=1}^{n} \xi_{i} \frac{\partial}{\partial \xi_{i}} \in H$ is diagonalizable and has finitely many eigenvalues.

Hence there exists a maximal weight $\lambda$. The eigenspace $V_{\lambda}$ is an irreducible module over $C_{L}(H)$. In the cases $W, S, \mathrm{CK}(6)$ the centralizer $C_{L}(H) \cong \operatorname{Vir}+\mathbb{C}\left[t, t^{-1}\right] \otimes H$ is a split extension of Vir. In the case $K$ we have $\operatorname{Rad} C_{L}(H) V_{\lambda}=(0)$, hence $V_{\lambda}$ is an irreducible module over $C_{L}(H) / \operatorname{Rad} C_{L}(H) \cong \operatorname{Vir}+\mathbb{C}\left[t, t^{-1}\right] \otimes H$ for $K(2 n)$ or $\mathfrak{g}+\mathfrak{g} \otimes H$, where $\mathfrak{g}$ is the Neveu-Schwarz or Ramond superalgebra for $K(2 n+1)$.

In any case, we conclude that there exist scalars $\alpha, \beta \in \mathbb{C}$ such that

$$
V \cong V(\lambda \mid \alpha, \beta)
$$

Now we will address the question for which $\lambda \in H^{*} ; \alpha, \beta \in \mathbb{C}$ the module $V(\lambda \mid \alpha, \beta)$ is a Harish-Chandra module?

We will discuss each case $W, S, K, \mathrm{CK}(6)$ separately.

4.3 $L=W(1: n), n \geq 2$.

For each $1 \leq i \neq j \leq n$ the elements

$$
\xi_{i} \frac{\partial}{\partial \xi_{j}}, \xi_{i} \frac{\partial}{\partial \xi_{i}}-\xi_{j} \frac{\partial}{\partial \xi_{j}}, \xi_{j} \frac{\partial}{\partial \xi_{i}}
$$

form an $\mathfrak{s l}_{2}$-triple. It implies that for the module $V(\lambda \mid \alpha, \beta)$ to be Harish-Chandra the highest weight $\lambda$ has to be dominant, i.e.

$$
\left\langle\lambda \mid \xi_{i} \frac{\partial}{\partial \xi_{i}}-\xi_{j} \frac{\partial}{\partial \xi_{j}}\right\rangle \in \mathbb{Z}_{\geq 0}
$$

for $1 \leq j<i \leq n$.

Theorem 4.3 For an arbitrary dominant functional $\lambda$ and arbitrary scalars $\alpha, \beta \in \mathbb{C}$ the module $V(\lambda \mid \alpha, \beta)$ is a Harish-Chandra module.

Thus graded irreducible $W(1: n)$-modules from the category $\mathcal{S}$ are parametrized by 3 continuous parameters 


$$
\alpha \in \mathbb{C} / \mathbb{Z}, \quad \beta \in \mathbb{C}, \quad \gamma=\left\langle\lambda \mid \sum_{i=1}^{n} \xi_{i} \frac{\partial}{\partial \xi_{i}}\right\rangle \in \mathbb{C},
$$

and $n-1$ discrete parameters

$$
\left\langle\lambda \mid \xi_{i+1} \frac{\partial}{\partial \xi_{i+1}}-\xi_{i} \frac{\partial}{\partial \xi_{i}}\right\rangle, \quad 1 \leq i \leq n-1 .
$$

4.4 $L=S(n, \gamma), n \geq 2$.

We still call a functional $\lambda: H^{(0)} \rightarrow \mathbb{C}$ dominant if

$$
\left\langle\lambda \mid \xi_{i} \frac{\partial}{\partial \xi_{i}}-\xi_{j} \frac{\partial}{\partial \xi_{j}}\right\rangle \in \mathbb{Z}_{\geq 0}, \quad 1 \leq j<i \leq n .
$$

Thus the restriction of a dominant functional on $H$ (in the case of $W(1: n)$ ) to $H^{(0)}$ is dominant.

Clearly, for a module $V(\lambda \mid \alpha, \beta)$ to be Harish-Chandra the functional $\lambda$ has to be dominant.

Theorem 4.4 For an arbitrary dominant functional $\lambda \in\left(H^{(0)}\right)^{*}$ and arbitrary scalars $\alpha, \beta \in \mathbb{C}$ the $S(n, \gamma)$-module $V(\lambda \mid \alpha, \beta)$ is a Harish-Chandra module.

4.5 $L=K(n), n \geq 2$.

We will consider separately the cases $n=2, n=3$ and $n \geq 4$.

4.5.1 $L=K(2)$. The Cartan subalgebra $H=\mathbb{C} \xi_{1} \eta_{1}$ in this case is one dimensional. The action of the element $\xi_{1} \eta_{1}$ on $V$ has finitely many eigenvalues. If $\lambda$ is the maximal eigenvalue, then $V \cong V(\lambda \mid \alpha, \beta)$ for some $\alpha, \beta \in \mathbb{C}$. For any $\alpha, \beta, \lambda \in \mathbb{C}$ the module $V(\lambda \mid \alpha, \beta)$ is a Harish-Chandra module over $L=K(2)$.

4.5.2 $L=K(3)=\left(\mathbb{C}\left[t, t^{-1}, \xi, \eta, \mu\right]\right.$, [, ]). The Cartan subalgebra $H=\mathbb{C} \xi \eta$ is still one dimensional, but now it is embeddable in $\mathfrak{s l}_{2}(\mathbb{C})$. The elements $2 \xi \mu, 2 \xi \eta, \mu \eta$ form an $\mathfrak{s l}_{2}$-triple. Let $V \in \mathcal{S}$ be an irreducible $L$-module. The eigenvalue $\lambda$ of the action of $2 \xi \eta$ on $V$ is an integer. Let $V=V(\lambda \mid \alpha, \beta)$ for some $\alpha, \beta \in \mathbb{C}$.

Theorem 4.2.2 For $\lambda=0$ and arbitrary scalars $\alpha, \beta \in \mathbb{C}$ the module $V(0 \mid \alpha, \beta)$ is never Harish-Chandra. For $\lambda=1$ the module $V(1 \mid \alpha, \beta)$ is Harish-Chandra if and only if $\alpha=-\frac{1}{4}$. For $\lambda \geq 2$ the module $V(\lambda \mid \alpha, \beta)$ is Harish-Chandra for all $\alpha, \beta \in \mathbb{C}$.

The superalgebras $K(3)$ (there are two of them), are the TKK constructions of Kantor doubles of brackets on $\mathbb{C}\left[t, t^{-1}\right]$. The case $\lambda=1$ corresponds to one-sided modules over these Jordan superalgebras. A description of such modules was given in [18].

4.5.3 $L=K(2 n)$ or $K(2 n+1), n \geq 2$. Denote $\lambda_{i}=\left\langle\lambda \mid \xi_{i} \mu_{i}\right\rangle, 1 \leq i \leq n$. For any $1 \leq j<i \leq n$ the triples $\xi_{i} \eta_{j}, \xi_{i} \eta_{i}-\xi_{j} \eta_{j}, \xi_{j} \eta_{i}$ and $\xi_{i} \xi_{j}, \xi_{i} \eta_{i}+\xi_{j} \eta_{j}, \eta_{j} \eta_{i}$ are $\mathfrak{s l}_{2}$-triples. In the odd case the triples $\mu \xi_{i}, 2 \xi_{i} \eta_{i}, 2 \eta_{i} \mu, 1 \leq i \leq n$, are also $\mathfrak{s l}_{2}$-triples. 
If $V(\lambda \mid \alpha, \beta)$ is a Harish-Chandra module then $\lambda_{i}-\lambda_{j}, \lambda_{i}+\lambda_{j} \in \mathbb{Z}_{\geq 0}$. Hence either $\lambda_{1}, \ldots, \lambda_{n} \in \mathbb{Z}, \lambda_{1} \leq \cdots \leq \lambda_{n}$, or $\lambda_{1}, \ldots, \lambda_{n} \in \frac{1}{2}+\mathbb{Z}, \lambda_{1} \leq \cdots \leq \bar{\lambda}_{n}$, and in both cases $\lambda_{1}+\lambda_{2} \in \mathbb{Z}_{\geq 0}$. We call such functionals dominant.

Theorem 4.2.3 (1) Let $\lambda$ be a dominant functional, $\alpha, \beta \in \mathbb{C}$. The module $V(\lambda \mid \alpha, \beta)$ over $K(2 n), n \geq 2$ is a Harish-Chandra module if and only if $\lambda_{1}+\lambda_{2} \geq 2$.

(2) The module $V(\bar{\lambda} \mid \alpha, \beta)$ over $K(2 n+1), n \geq 2$, is a Harish-Chandra module if and only if $\lambda_{1} \geq 2$.

4.6 $L=C K(6)$.

The Cartan subalgebra $H$ in this case is spanned by $h_{\omega_{i}-\omega_{j}}, 1 \leq i \leq 4$ (see Sect. 3). If $V(\lambda \mid \alpha, \beta)$ is a Harish-Chandra module then $\left\langle\lambda \mid h_{\omega_{i}-\omega_{j}}\right\rangle \in \mathbb{Z}_{\geq 0}$ whenever $f\left(\omega_{i}-\omega_{j}\right)>0$.

Let $\lambda$ be a dominant weight and let $m=\left\langle\lambda \mid h_{\omega_{1}-\omega_{3}}\right\rangle$. Let $\alpha, \beta \in \mathbb{C}$.

Theorem 4.6 If $m=0$ aand $\alpha, \beta \in \mathbb{C}$ then $V(\lambda \mid \alpha, \beta)$ is never a Harish-Chandra module. If $m=1$ then $V(\lambda \mid \alpha, \beta)$ is a Harish-Chandra module if and only if $\left\langle\lambda \mid h_{\omega_{2}-\omega_{3}}\right\rangle=0, \beta=-1$. If $m \geq 2$ then $V(\lambda \mid \alpha, \beta)$ is a Harish-Chandra module for any $\alpha, \beta \in \mathbb{C}$.

Remark The numbers $\lambda_{1}+\lambda_{2}$ in the case $K(2 n), n \geq 2, \lambda_{1}$ in the case $K(2 n+1)$ and $\left\langle\lambda \mid h_{\omega_{1}-\omega_{3}}\right\rangle$ in the case $\mathrm{CK}(6)$ are spectral gaps that gained prominence in the study of expanders (see [17]).

\section{More open questions}

Let $L$ be one of the superconformal algebras discussed in Sects. 3, 4. For every weight $\mu$ of an $L$-module $V(\lambda \mid \alpha, \beta)$ the weight space $V_{\mu}$ is a $\mathbb{C}\left[t, t^{-1}\right]$-module of finite rank that we denote by $\operatorname{rank}\left(V_{\mu}\right)$.

Question 4 Find a formula for the character

$$
\operatorname{ch}(V)=\sum_{\mu} \operatorname{rank}\left(V_{\mu}\right) e^{\mu} .
$$

The associative commutative superalgebras $\Lambda(m: n)$ and $\Lambda\left(m_{1}: m_{2}: n\right), m_{1}+$ $m_{2}=m$, and the related Lie superalgebras of types $W, S, H, K$ are graded by the group $\mathbb{Z}^{m}$. A $\mathbb{Z}^{m}$-graded module

$$
V=\sum_{\alpha \in \mathbb{Z}^{m}} V_{\alpha}
$$

is said to be a Harish-Chandra module if $\operatorname{dim} V_{\alpha}<\infty$ for all $\alpha \in \mathbb{Z}^{m}$. In [3] Billig and Futorny classified irreducible Harish-Chandra modules over $W(m: 0)$. On the other hand Bakalov et al. [2] developed a theory of pseudoalgebras that, in particular, applies to formal distributions in several variables. 
Let $L$ be one of the superalgebras $W\left(m_{1}: m_{2}: n\right), S\left(m_{1}: m_{2}: n, \alpha\right), H\left(m_{1}\right.$ : $\left.m_{2}: n\right), K\left(m_{1}: m_{2}: n\right), m_{1}+m_{2}=m$. The dimensions of all nonzero homogeneous components $L_{\alpha}, \alpha \in \mathbb{Z}^{m}$, are equal to $d$, and in each $L_{\alpha}$ we can choose a standard basis $e_{\alpha 1}, \ldots, e_{\alpha d}$.

We say that an $L$-module $V=\sum_{\alpha \in \mathbb{Z}^{m}} V_{\alpha}$ satisfies the Polynomiality Assumption if the dimensions of all nonzero homogeneous components $V_{\alpha}, \alpha \in \mathbb{Z}^{m}$, are equal to $q$ and in each $V_{\alpha} \neq 0$ we can choose a basis $v_{\alpha 1}, \ldots, v_{\alpha q}$ such that the $d q^{2}$ structural constant functions $\gamma_{i j}^{r}(\alpha, \beta), 1 \leq i \leq d, 1 \leq j, r \leq q$

$$
e_{\alpha i} v_{\beta j}=\sum_{r=1}^{q} \gamma_{i j}^{r}(\alpha, \beta) v_{\alpha+\beta, r}
$$

are polynomials in $\alpha_{1}, \ldots, \alpha_{m}, \beta_{1}, \ldots, \beta_{m}$.

Conjecture Let $V$ be an irreducible Harish-Chandra module over L. Then either $V$ is a "highest weight module" (see [3]) or V satisfies the Polynomiality Assumption.

Remark 1 The Polynomiality Assumption implies that the description of irreducible (not highest weight) Harish-Chandra modules over $L=W(m: n), S(m: n, \alpha)$, $H(m: n)$ or $K(m: n)$ should be "parallel" to the description of conformal modules of finite type in [2].

Remark 2 Let $L$ be one of the superalgebras $W\left(m_{1}: m_{2}: n\right), S\left(m_{1}: m_{2}: n, \alpha\right)$, $H\left(m_{1}: m_{2}: n\right), K\left(m_{1}: m_{2}: n\right), m_{1}+m_{2}=m$. The Polynomiality Assumption implies that for $m_{2} \geq 1$ the action of $L$ on $V$ can be extended to an action of the corresponding superalgebra $W(m: n), S(m: n, \alpha), H(m: n)$ or $K(m: n)$.

Acknowledgements The first author has been partially supported by the Projects MTM 2017-83506-C22-P from the Ministry of Economy of Spain and FC-GRUPIN-IDI/2018/000193 from the Principado de Asturias, the second author gratefully acknowledges the support by the NSF Grant DMS 1601920.

Author contributions CM, EZ designed research, performed research, and wrote the paper.

\section{Compliance with ethical standards}

Conflict of interest The authors declare that they have no conflict of interest.

\section{References}

1. Ademollo, M., et al.: Supersymmetric strings and color confinement. Phys. Lett. 62B, 105-110 (1976)

2. Bakalov, B., D’Andrea, A., Kac, V.G.: Theory of finite pseudoalgebras. Adv. Math. 162(1), 1-140 (2001)

3. Billig, Y., Futorny, V.: Classification of irreducible representations of Lie algebra of vector fields on a torus. J. Reine Angew. Math. 720, 199-216 (2016)

4. Cantarini, N., Kac, V.G.: Classification of linearly compact simple Jordan and generalized Poisson superalgebras. J. Algebra 313(1), 100-124 (2007)

5. Cheng, S.-J., Kac, V.G.: A new $N=6$ superconformal algebra. Commun. Math. Phys. 186(1), 219-231 (1997)

6. Dong, C.: Vertex algebras associated with even lattices. J. Algebra 161(1), 245-265 (1993) 
7. Fattori, D., Kac, V.G.: Classification of finite simple Lie conformal superalgebras. J. Algebra 258(1), 23-59 (2002). (Special issue in celebration of Claudio Procesi's 60th birthday)

8. Grozman, P., Leites, D., Shchepochkina, I.: Lie superalgebras of string theories. Acta Math. Vietnam 26(1), 27-63 (2001)

9. Kac, V.G.: Lie superalgebras. Adv. Math. 26(1), 8-96 (1977)

10. Kac, V.G., Martinez, C., Zelmanov, E.: Graded simple Jordan superalgebras of growth one. Mem. Am. Math. Soc. 150(711), x+140 (2001)

11. Kac, V.G. van de Leur, J.W.: On classification of superconformal algebras. In: Strings '88 (College Park, MD, 1988), pp. 77-106. World Scientific Publishing, Teaneck (1989)

12. Kac, V.: Vertex Algebras for Beginners, University Lecture Series, vol. 10. American Mathematical Society, Providence (1997)

13. Kantor, I.L.: Certain generalizations of Jordan algebras. Trudy Sem. Vektor. Tenzor. Anal. 16, 407-499 (1972)

14. Kantor, I.L.: Connection Between Poisson Brackets and Jordan and Lie Superalgebras, Lie Theory, Differential Equations and Representation Theory (Montreal, PQ, 1989), pp. 213-225. University of Montréal, Montreal (1990)

15. Kaplansky, I., Santharoubane, L.J.: Harish-Chandra modules over the Virasoro algebra. In: InfiniteDimensional Groups with Applications (Berkeley, California, 1984), Mathematical Sciences Research Institute Publications, vol. 4, pp. 217-231. Springer, New York (1985)

16. Koecher, M.: Imbedding of Jordan algebras into Lie algebras. I. Am. J. Math. 89, 787-816 (1967)

17. Lubotzky, A.: Expander graphs in pure and applied mathematics. Bull. Am. Math. Soc. (N.S.) 49(1), 113-162 (2012)

18. Martínez, C., Zelmanov, E.: Specializations of Jordan superalgebras. Can. Math. Bull. 45(4), 653-671 (2002). (Dedicated to Robert V. Moody)

19. Martínez, C., Zelmanov, E.: Representation theory of Jordan superalgebras. I. Trans. Am. Math. Soc. 362(2), 815-846 (2010)

20. Martí nez, C., Zelmanov, E.I.: Lie superalgebras graded by $P(n)$ and $Q(n)$. Proc. Natl. Acad. Sci. USA 100(14), 8130-8137 (2003)

21. Martin, C., Piard, A.: Classification of the indecomposable bounded admissible modules over the virasoro lie algebra with weightspaces of dimension not exceeding two. Commun. Math. Phys. 150(3), 465-493 (1992)

22. Martinez, C., Zelmanov, E.: Simple finite-dimensional Jordan superalgebras of prime characteristic. J. Algebra 236(2), 575-629 (2001)

23. Mathieu, O.: Classification of Harish-Chandra modules over the Virasoro Lie algebra. Invent. Math. 107(2), 225-234 (1992)

24. Neveu, A., Schwarz, J.H.: Factorizable dual model of pions. Nucl. Phys. B 31(1), 86-112 (1971)

25. Ramond, P.: Dual theory for free fermions. Phys. Rev. D (3) 3, 2415-2418 (1971)

26. Schoutens, K.: A nonlinear representation of the $d=2 \mathrm{SO}(4)$ extended superconformal algebra. Phys. Lett. B 194, 75-80 (1987)

27. Schwimmer, A., Seiberg, N.: Comments on the $\mathrm{n}=2,3,4$ superconformal algebras in two dimensions. Phys. Lett. B 184(2), 191-196 (1987)

28. Tits, J.: Une classe d'algèbres de Lie en relation avec les algèbres de Jordan. Nederl. Akad. Wetensch. Proc. Ser. A 65 = Indag. Math. 24, 530-535 (1962)

29. Tits, J.: Algèbres alternatives, algèbres de Jordan et algèbres de Lie exceptionnelles. I. Construction. Nederl. Akad. Wetensch. Proc. Ser. A 69 = Indag. Math. 28, 223-237 (1966)

Publisher's Note Springer Nature remains neutral with regard to jurisdictional claims in published maps and institutional affiliations. 